\title{
Childhood cancer incidence in Georgia: Descriptive epidemiology, geographic trends, and disparities in insurance coverage and health care access
}

\author{
Florence A. Kanu, MPH, ${ }^{1}$ Sara Wagner Robb, $\mathrm{PhD},{ }^{2}$ and Rosemary Corriero ${ }^{3}$
}

${ }^{1} \mathrm{PhD}$ Student, College of Public Health, Department of Epidemiology and Biostatistics, University of Georgia, Athens, GA; ${ }^{2}$ Assistant Professor, College of Public Health, Department of Epidemiology and Biostatistics, University of Georgia, Athens, GA; and ${ }^{3} \mathrm{MPH}$ Student, College of Public Health, Department of Health Promotion and Behavior, University of Georgia, Athens, GA

\begin{abstract}
Background: Limited research has been conducted concerning childhood cancer (CC) incidence in Georgia, which is a leading cause of death for children in the US. The purpose of this study was to determine if county-level CC incidence rates differed by geography or race and if health care access disparities exist.
\end{abstract}

Methods: Incidence data were obtained from the Georgia Comprehensive Cancer Registry for 2000-2011. Age-adjusted incidence rates per 100,000 were analyzed by sex, race, and county. Hotspots and coldspots of CC incidence were analyzed using the Getis-Ord GI* statistic. Health care access data for children under 19 were obtained using US Census Bureau's Small Area Health Insurance Estimates for 2011. Georgia's three children's oncology group (COG) treatment facilities with 40-mile buffer zones were geographically overlaid with $\mathrm{CC}$ incidence rate maps and health insurance maps using Geographic Information Systems (GIS).

Results: For leukemia and central nervous system cancers, incidence rates were significantly different between Whites [7.8, 95\% confidence interval (CI) $(7.4,8.2)]$ and Blacks $[5.2,95 \%$ CI $(4.8,5.6)]$. Statistical hotspots of CC were observed in north Georgia. A lower percentage of insurance coverage among children was observed in southeast GA. Approximately $25 \%$ of Georgia counties that were not within a COG buffer had a higher percentage of children who were uninsured (mean \pm SD: $10.28 \% \pm$ $1.86 \%)$.

Conclusion: Higher CC incidence rates and disparities in access to care were evident in north Georgia. Future research is needed in these geographies to investigate potential risk factors associated with CC incidence patterns and racial differences in Georgia.

Key words: childhood cancer; epidemiology; GIS; racial disparities; access to care; health insurance coverage

\section{INTRODUCTION}

With an estimated 15,780 new cases diagnosed each year in the United States (US), childhood cancer (CC) is among the top causes of death for children ages 0 to 19 (American Childhood Cancer Organization, 2015; National Cancer Institute, 2013). Unlike cancer that occurs in adults, the etiology of most CC cases, the most common types of which are leukemia and central nervous system (CNS) cancers (McNamara C, 2012; National Cancer Institute, 2014a), is often unknown. Moreover, early detection of $\mathrm{CC}$ can be challenging since initial symptoms are generally non-specific (National Cancer Institute, 2013). According to the Georgia Comprehensive Cancer Registry (GCCR), the CC incidence rate for all cancer types was 16.4 per 100,000 in Georgia (GA) for the year 2011, which is slightly lower than the national 2011 average of 17.9 per 100,000 (Centers for Disease Control and Prevention, 2014). While the incidence of CC may be slightly lower in GA compared to the US, there are counties within GA with incidence rates that are higher than that of the nation. For instance, Oconee County, located in northeast GA, had an annual age-adjusted CC incidence rate of 35.0 per 100,000 from 2007 to 2011 , which is higher than the national rate and almost double GA's statewide $\mathrm{CC}$ incidence rate (National Cancer Institute, 2014b). Overall, a total of 20 counties in GA exceeded the nation's overall CC incidence rate from 2007 to 2011 (National Cancer Institute, 2014b), suggesting that epidemiologic research of CC in GA should be a priority.

To date, limited research regarding $\mathrm{CC}$ in $\mathrm{GA}$ has been conducted. A recent report by the GCCR indicated an increase in CC mortality in GA since 2002 for reasons that are unknown (McNamara C, 2012). Based on their findings, the GCCR acknowledges $\mathrm{CC}$ as an important public health issue with appropriate access to care being a main priority in the fight against CC (National Cancer Institute, 2014b). Scientific descriptions of $\mathrm{CC}$ in GA in the peer-reviewed literature are needed to provide insight into potential etiologic mechanisms and support the generation of more tailored analytic epidemiologic studies. 
White children are about three times more likely to be diagnosed with $\mathrm{CC}$ and are about two times more likely to die from $\mathrm{CC}$ as compared to children of other racial subgroups (Howell, Ward, Austin, Young, \& Woods, 2007). Despite this, limited research on CC among Black children has been conducted, which is important in fully describing the burden that CC places on the entire population. According to 2013 census data, GA has a relatively high percentage of Black residents with a composition of approximately $62.5 \%$ White and $31.4 \%$ Black, while the US is approximately $77.7 \%$ White and $13.2 \%$ Black (U.S. Census Bureau, 2015c), which may facilitate racial comparisons of disease further supporting the investigation of CC in GA.

Providing access to age-appropriate cancer treatment to children and adolescents upon cancer diagnosis is ideal. It is recognized that under-utilization of pediatric oncology services may be partially responsible for the lack of long-term survival among this population (Bleyer, 2002). In GA, there are currently only three Children Oncology Group (COG) treatment facilities, located in north, northeast, and southeast GA, which provide residents with specialized $\mathrm{CC}$ treatment (Children's Oncology Group, 2014). The COG's in GA are few in number relative to many other states and their distribution is not equal throughout the state, which may create access to care issues for residents in specific areas of GA. The distribution of COG centers combined with potential variations in child health insurance coverage may be important contributing factors to disparities in CC outcomes in GA.

\section{PURPOSE}

Given the lack of epidemiological descriptions of CC in GA, the purpose of the paper was to describe $\mathrm{CC}$ in the state of GA, with an emphasis on describing geographic trends and disparities in health care access, as indicated by COG center distribution and county-level child health insurance coverage. This study analyzed data for White and Black children who were diagnosed with CC before age 20 during 2000-2011 in GA.

\section{METHODS}

CC incidence data were obtained from the GCCR for the years 2000-2011. The GCCR is a statewide population-based cancer registry that has been gathering information on all cancer cases identified among GA residents since 1995 (Georgia Department of Public Health, n.d.). The goal of the GCCR is to provide cancer incidence and mortality data to cancer control programs in order to aid in developing strategies and evaluating program effectiveness (Georgia Department of Public Health, n.d.). GCCR is part of the Surveillance, Epidemiology, and End Results (SEER) Program, which is a respected source of information on cancer incidence and survival in the US and is gold certified with high ratings for data quality and representativeness (National Cancer Institute, n.d.). Directly standardized age-adjusted CC incidence rates per 100,000 population were obtained for analysis (SEER*Stat software version 8.2.1; Bethesda, MD).

Childhood cancer incidence rates were analyzed by sex, race (White vs. Black), and county $(\mathrm{N}=159)$. Statistical significance of $\mathrm{CC}$ incidence rates between sexes, races, and counties was determined based on non-overlapping $95 \%$ confidence intervals (CI). All maps included combined CC incidence rates of leukemia and CNS cancers.

Inequalities in access to care for children with cancer were also evaluated by race and county. Access to care data were obtained from the US Census Bureau's Small Area Health Insurance Estimates (SAHIE) program for the year 2011. The SAHIE program provides single-year estimates of health insurance coverage for every US County (U.S. Census Bureau, 2015a). Percent of children under 19 years of age without health insurance was used as an index for poor access to care. This measure was evaluated geographically by assigning each county with a corresponding uninsured percentage of children under 19 years of age, which represented potential statewide access to care disparities.

Additionally, GA's three COG treatment facilities were geographically overlaid with the uninsured percentage variable onto each $\mathrm{CC}$ incidence rate map to examine if counties within a 40-mile radius of a COG center had a greater number of children with health insurance. Each COG buffer was created using the Buffer Analysis Tool in Geographic Information Systems (GIS) (ArcMap software, version 10.2.2; ESRI, Redlands, CA). The 40-mile radius (i.e., COG buffer) was chosen based on the criteria used by the American Cancer Society's Hope Lodge. Each Hope Lodge offers cancer patients and their families a free place to stay when their best hope for effective treatment may be in another city. Patients who have to travel more than 40 miles away from their treatment center are eligible to participate with the Hope Lodge in their area (American Cancer Society, 2015). For the purpose of this analysis, if the geographic centroid of a county fell within the 40-mile radius, it was counted as inside the COG buffer. The number of counties that fell within a COG buffer were counted and compared to the number of counties that were outside of the identified COG buffers.

\section{Statistical Analysis}

County-level maps of $\mathrm{CC}$ incidence rates in GA were created using GIS. Maps were generated separately by race. CC incidence rates were divided into quartiles on each map, the higher the CC incidence rate, the darker the color. Quartile category ranges differed on each map depending on the racial group examined. Hotspots and coldspots of CC incidence rates at the county level were evaluated using the Getis-Ord Gi* statistic in GIS. The Getis-Ord Gi* statistic identifies clusters of counties with incidence rates higher (hotspots) or lower (coldspots) in magnitude than what is expected by random chance. Z-scores, indicating that neighboring counties have a correspondingly high or low $\mathrm{CC}$ incidence rate, and confidence intervals were generated for each county in GA to depict significant hotspots or coldspots of $\mathrm{CC}$ incidence rates. Z-scores near zero indicated no evident clustering of high or low incidence rates.

Access to health care was geographically represented using the percent of children without health insurance variable from the SAHIE program. Uninsured percentages were divided into quartiles and counties with greater percentages of uninsured children) (i.e. poorer access to care) were depicted with darker 
color gradations using circle symbols. These circular quantifications of health insurance coverage and the COG locations with corresponding 40-mile buffer zones were overlaid on the $\mathrm{CC}$ incidence rate maps to identify potential geographic patterns.

\section{RESULTS}

From 2000-2011, GA children (ages 0-19) experienced a combined age-adjusted cancer incidence rate of 15.7 per
100,000 for all cancer types. Specifically, for leukemia and CNS cancers, the age-adjusted incidence rate was 6.8 per 100,000 . Incidence rates for both leukemia and CNS cancers were slightly higher among males $(7.1$ per 100,000$)$ compared to females (6.5 per 100,000). Incidence rates for both leukemia and CNS cancers were significantly higher among White children $(7.8$ per $100,000,95 \%$ CI $(7.4,8.2)$ ) compared to Black children $(5.2$ per $100,000,95 \%$ CI $(4.8,5.6))$ from 2000-2011 (Table 1).

\begin{tabular}{|c|c|c|c|}
\hline & & 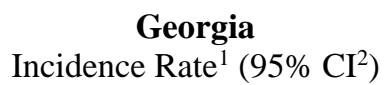 & $\begin{array}{c}\text { United States } \\
\text { Incidence Rate }{ }^{1}\left(95 \% \mathrm{CI}^{2}\right)\end{array}$ \\
\hline \multirow{4}{*}{$\begin{array}{c}\text { Cancer } \\
\text { Type }\end{array}$} & All Cancers ${ }^{3}$ & $15.7(15.3,16.2)$ & $17.0(16.9,17.2)$ \\
\hline & $\begin{array}{r}\text { Leukemia \& } \\
\text { CNS }^{4}\end{array}$ & $6.8(6.5,7.1)$ & $7.6(7.5,7.7)$ \\
\hline & Leukemia & $3.8(3.6,4.1)$ & $4.6(4.5,4.6)$ \\
\hline & $\mathrm{CNS}^{4}$ & $3.0(2.8,3.2)$ & $3.0(3.0,3.1)$ \\
\hline \multirow{2}{*}{$\operatorname{Sex}^{5}$} & Male & $7.1(6.7,7.6)$ & $8.2(8.1,8.3)$ \\
\hline & Female & $6.5(6.1,6.9)$ & $7.0(6.9,7.1)$ \\
\hline \multirow{2}{*}{ Race $^{5}$} & White & $7.8(7.4,8.2)$ & $8.3(8.2,8.4)$ \\
\hline & Black & $5.2(4.8,5.6)$ & $5.1(4.8,5.3)$ \\
\hline
\end{tabular}

${ }^{1}$ Incidence rates were directly standardized age-adjusted per 100,000 population.

${ }^{2} \mathrm{CIs}=$ Confidence Intervals

${ }^{3}$ All cancers $=$ All cancers sites reported in the SEER registry.

${ }^{4} \mathrm{CNS}=$ Central Nervous System cancers

${ }^{5}$ Reported rates are of leukemia and central nervous system cancers combined.

When age-adjusted incidence rates for leukemia and CNS cancers were examined geographically, low rates (lowest quartile; 0-11.6 per 100,000) were observed in the counties of southwest GA and high rates (highest quartile; 18.4-31.9 per 100,000 ) were observed in counties located in north GA (Fig. 1). There were no cases of CC in Clay (southwest GA), Glascock (east central GA), or Miller (southwest GA) counties. The highest $\mathrm{CC}$ incidence rates for leukemia and CNS cancers were observed in Twiggs and Randolph Counties
(31.1 per 100,000 and 31.9 per 100,000 , respectively) located in central and southwest GA, respectively (Fig. 1). When stratified by race, the geographic distribution of $\mathrm{CC}$ incidence rates changed slightly. Among White children, high CC incidence rates (highest quartile; 20.3-85.1 per 100,000) were present in central GA (Fig. 2). Among Black children, high rates (highest quartile; 16.2-205.3 per 100,000) of CC incidence were no longer clustered in north GA, but rather dispersed throughout the state (Fig. 3). 

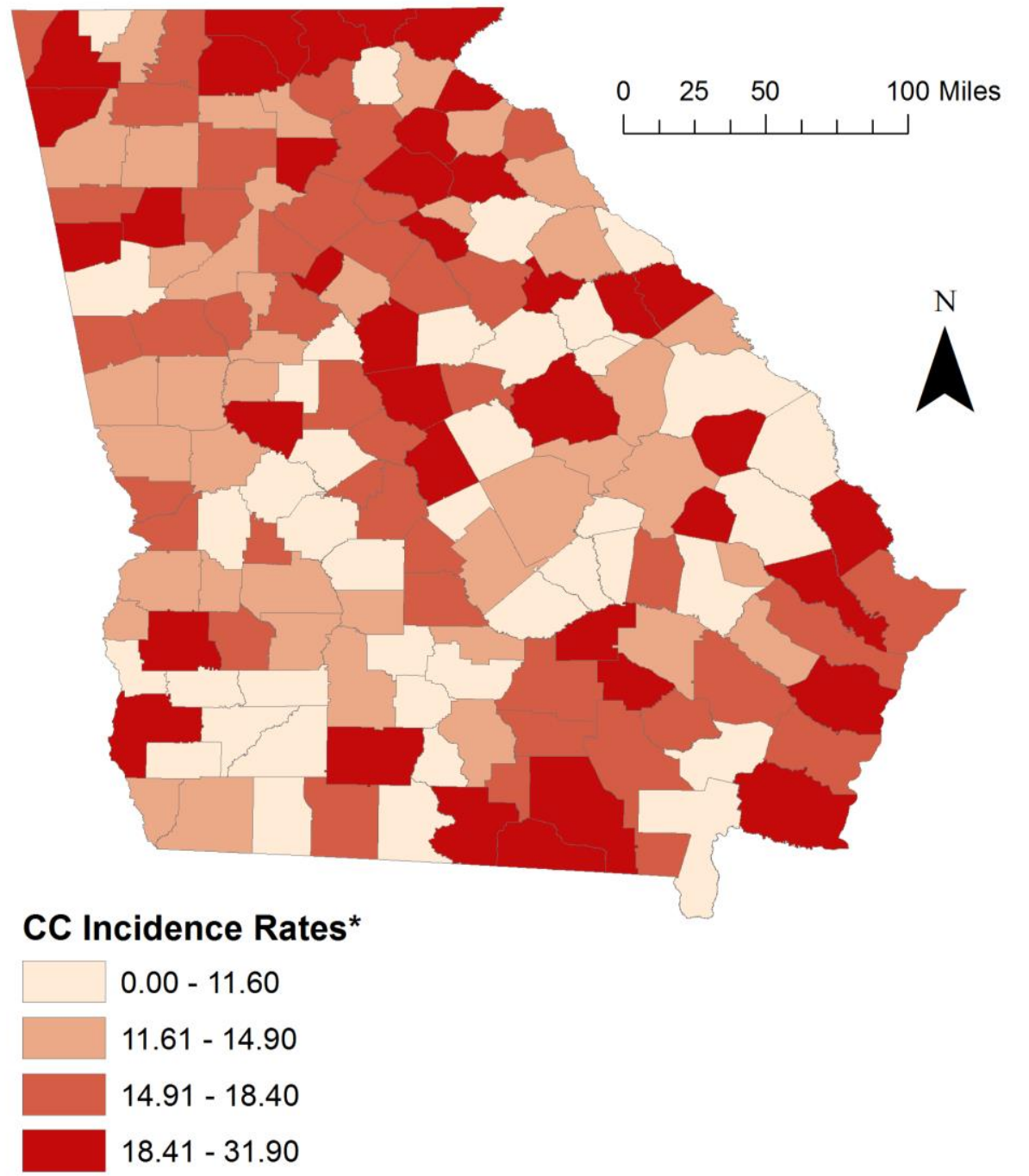

*Incidence rates (cases per 100,000 population per year) are age-adjusted to the 2000 US standard population (19 age groups: $<1,1-4, \ldots, 80-84,85+$ ). Rates are for invasive cancer only (except for bladder which is invasive and in situ) or unless otherwise specified. Rates calculated using SEER*Stat Population counts for denominators are based on Census populations as modified by NCl. The 1969-2012 US Population Data File is used for SEER and NPCR incidence rates.

Figure 1. CC incidence rates by county for both races, 2000-2011 


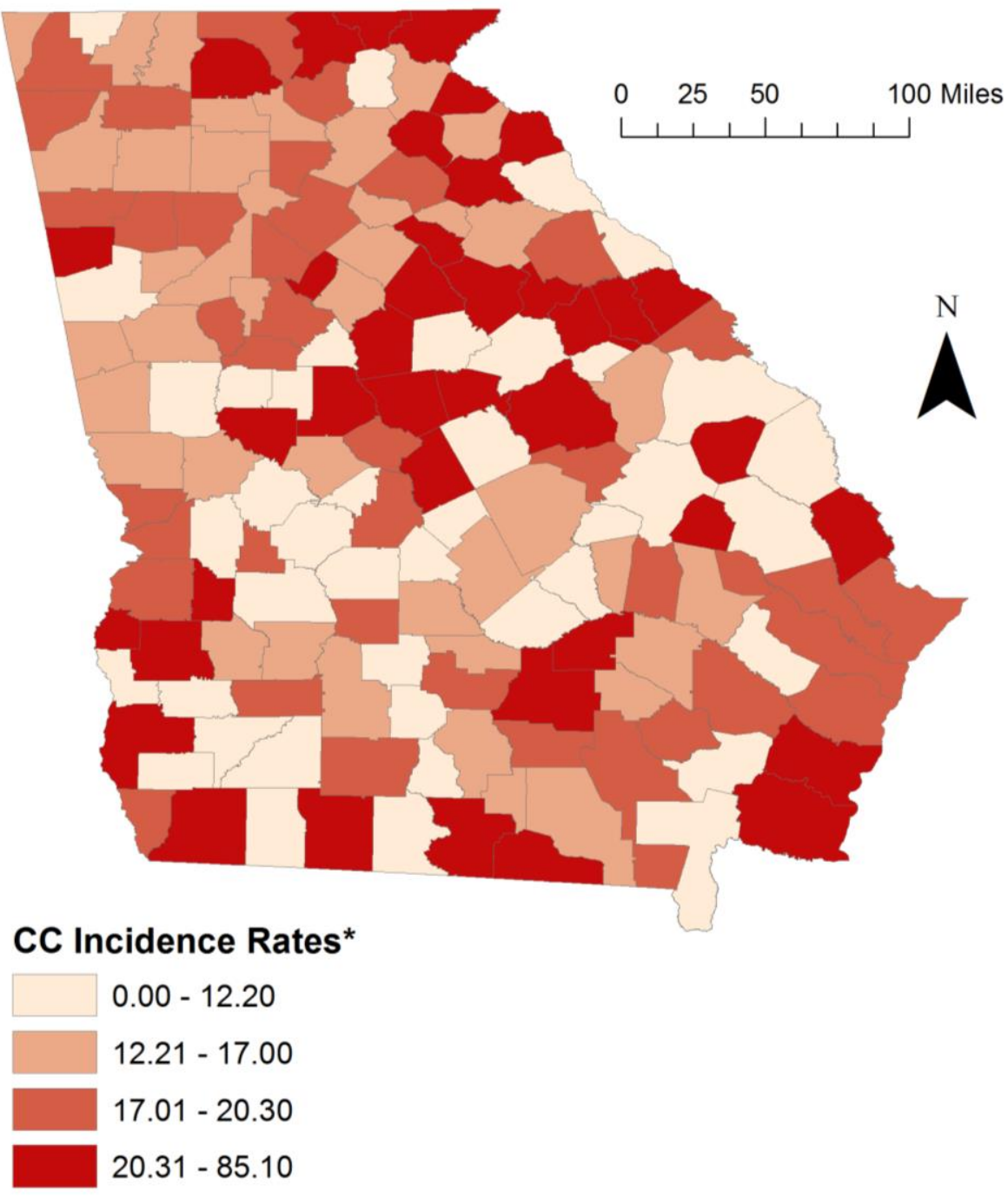

*Incidence rates (cases per 100,000 population per year) are age-adjusted to the 2000 US standard population (19 age groups: $<1,1-4, \ldots, 80-84,85+$ ). Rates are for invasive cancer only (except for bladder which is invasive and in situ) or unless otherwise specified. Rates calculated using SEER*Stat Population counts for denominators are based on Census populations as modified by NCl. The 1969-2012 US Population Data File is used for SEER and NPCR incidence rates

Figure 2. CC incidence rates of White children by county, 2000-2011 


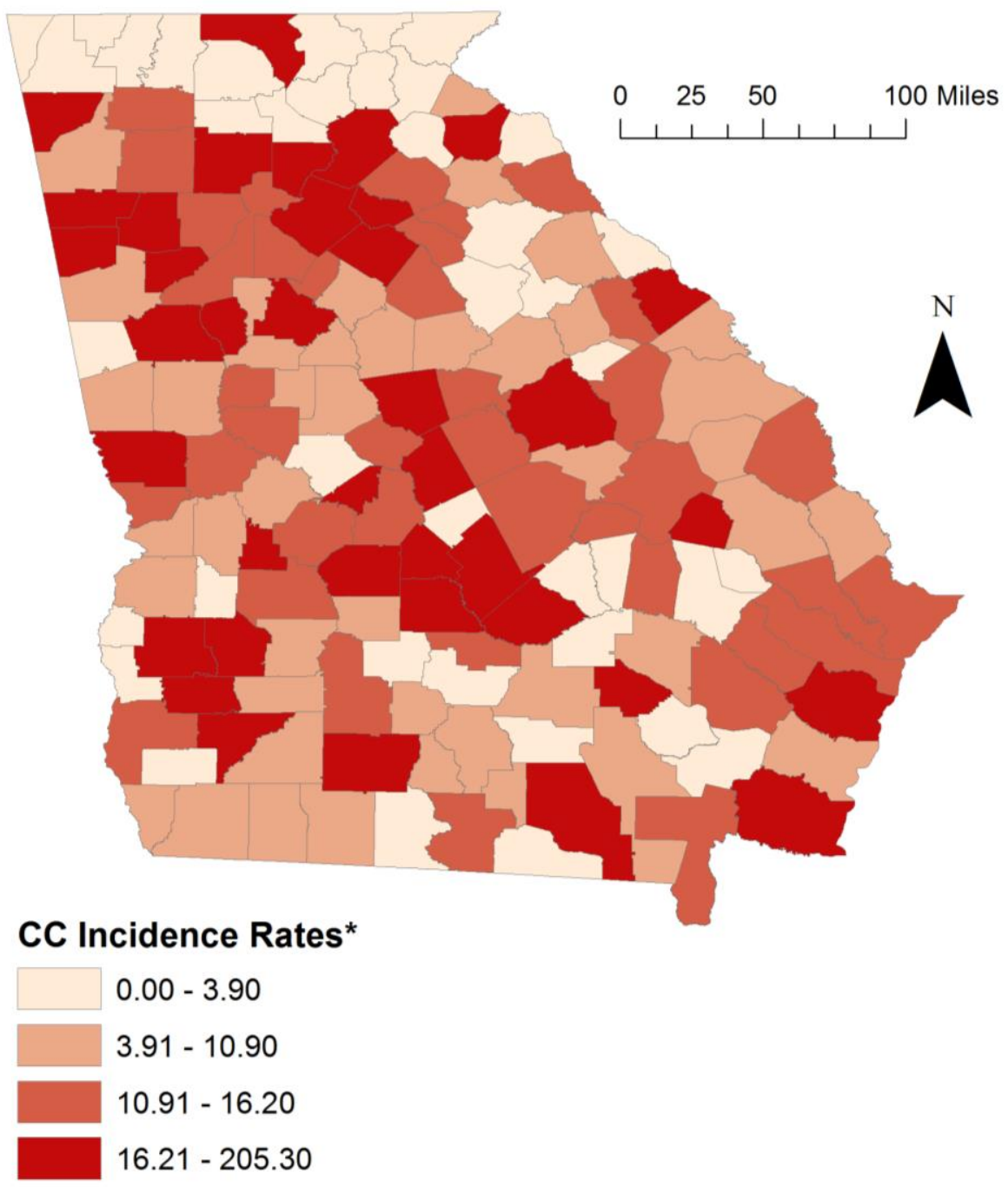

*Incidence rates (cases per 100,000 population per year) are age-adjusted to the 2000 US standard population (19 age groups: $<1,1-4, \ldots, 80-84,85+$ ). Rates are for invasive cancer only (except for bladder which is invasive and in situ) or unless otherwise specified. Rates calculated using SEER*Stat Population counts for denominators are based on Census populations as modified by NCl. The 1969-2012 US Population Data File is used for SEER and NPCR incidence rates.

Figure 3. CC incidence rates of Black children by county, 2000-2011 
When county-level hotspot analyses were completed, significant (95\% confidence or greater) hotspots of CC incidence rates, areas with high $\mathrm{CC}$ incidence, were primarily located in north GA for White and Black children combined (Fig. 4). The pattern shifted when stratified by race; significant hotspots of $\mathrm{CC}$ incidence rates among White children were primarily located in east central GA (Fig. 5). County-level hotspot analysis was not completed for Black children due to small sample size $(n=582 \mathrm{CC}$ cases). Significant coldspots, areas with low CC incidence, were located in southwest GA for both races (Fig. 4). This pattern was consistent when only White children were examined (Fig. 5).

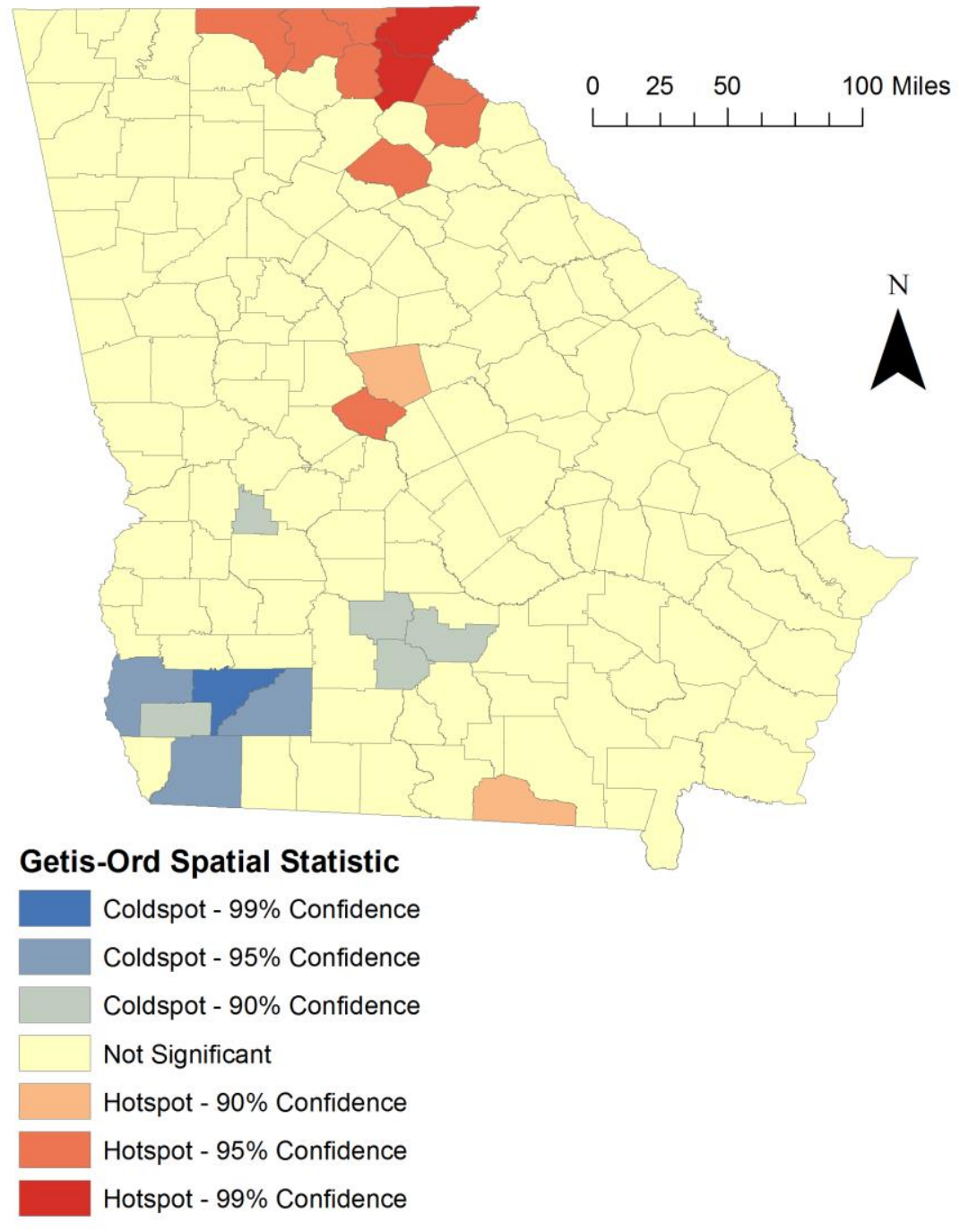

Figure 4. Getis-Ord Spatial Statistic for hotspot analysis of CC incidence rates for both races by county, $2000-2011$ 


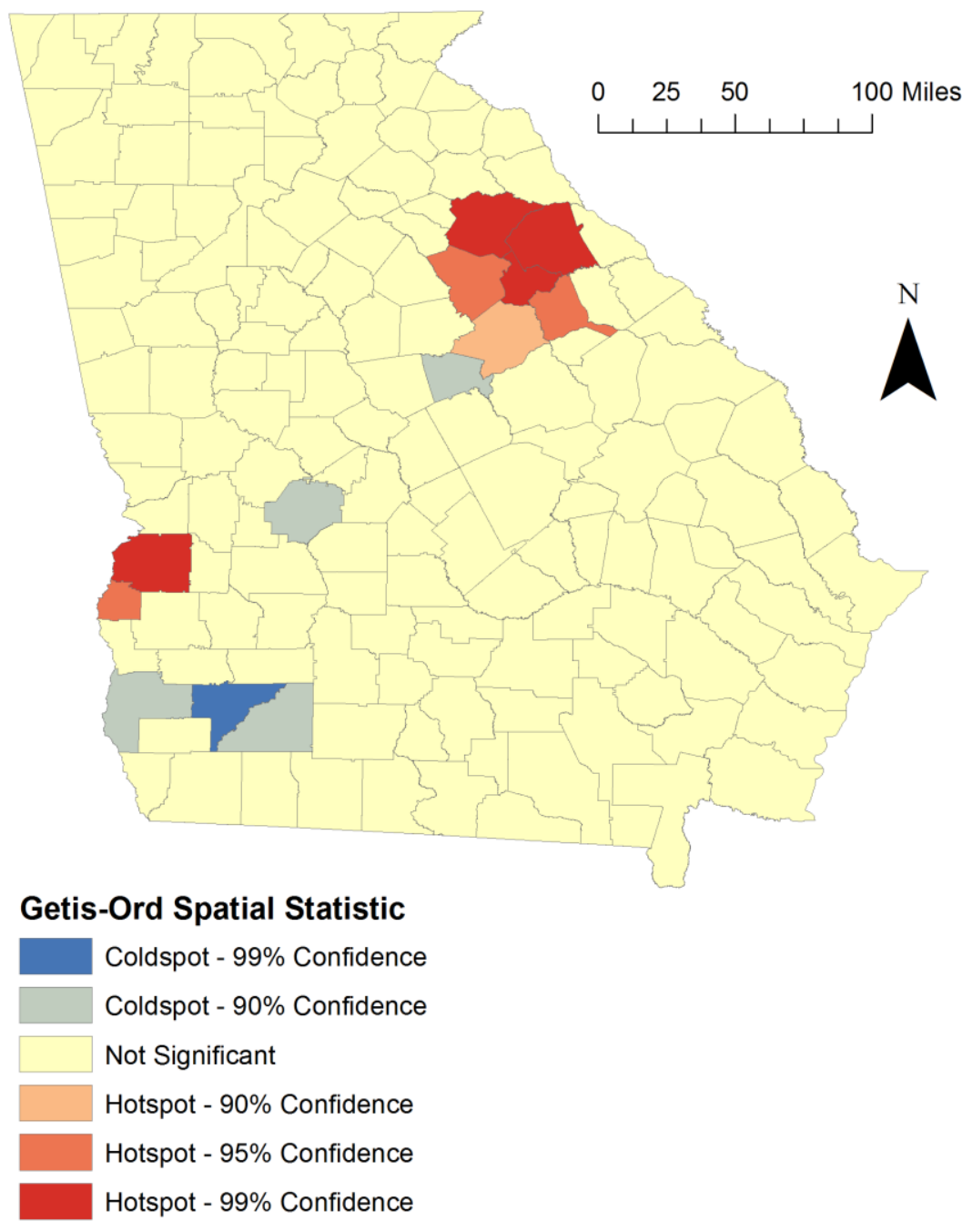

\section{Figure 5. Getis-Ord Spatial Statistic for hotspot analysis of CC incidence rates for White children by county, $2000-2011$}

According to the 2011 US Census Bureau's SAHIE program, the overall percent of GA children under 19 without health insurance was $10.1 \%$, with a minimum of $6.7 \%$ without health insurance in Chattahoochee County (located in west central GA) and a maximum of $19.1 \%$ without health insurance in Echols County (located in south GA). Greater proportions of uninsured children (i.e. poorer access to care percentages; highest quartile: $11.7 \%$ - 19.1\%) were observed throughout GA, mainly in southeast GA (Fig 6). Three counties (3/17;
17.6\%) within the Atlanta COG buffer were in the highest CC incidence rate quartile (highest quartile: 18.4-31.9) (Table 2). Within the Augusta COG buffer, two counties (2/8; 25\%) were in the highest $\mathrm{CC}$ incidence rate quartile. Additionally, two counties $(2 / 4 ; 50 \%)$ within the Savannah COG buffer were in the highest $\mathrm{CC}$ incidence rate quartile (Table 2). For both races, 33 counties $(33 / 130 ; 25.4 \%)$ were in the highest $\mathrm{CC}$ incidence rate quartile and were not contained in any $\mathrm{COG}$ buffer (Table 2). 


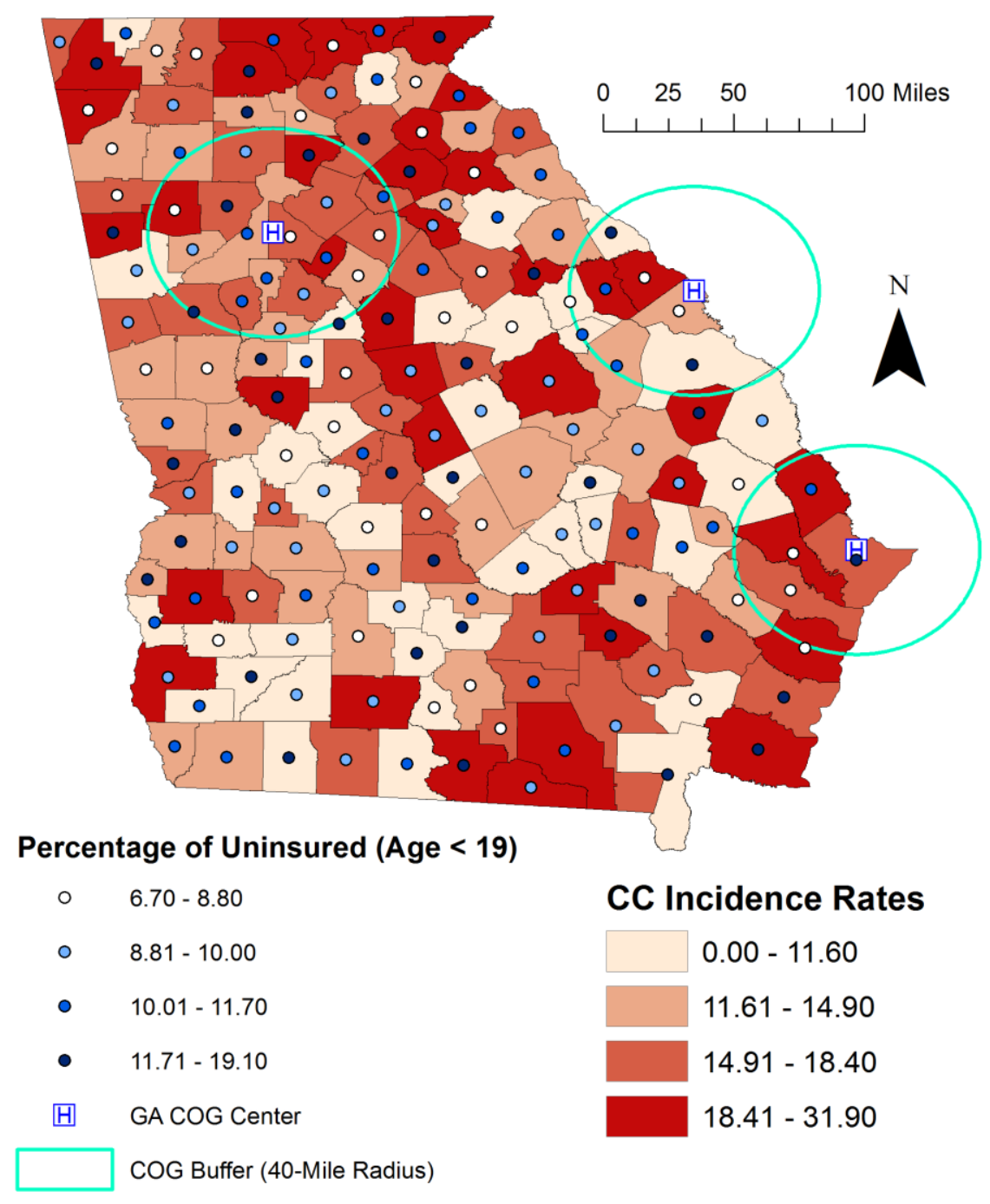

Figure 6. CC incidence rates and percentage of uninsured children under age 19 for both races by county with GA COGs 
Table 2. Summary of county-level ( $N=159)$ age-adjusted childhood cancer (ages 0 - 19 years) incidence rates from $2000-2011$ and percent of uninsured children (ages 0 - 19 years) by race and COG location in Georgia.

\begin{tabular}{|c|c|c|c|c|c|c|c|c|c|c|c|c|}
\hline & \multicolumn{3}{|c|}{$\begin{array}{l}\text { All Counties Outside COG Buffers }{ }^{1,2} \\
\qquad(n=130)\end{array}$} & \multicolumn{3}{|c|}{$\begin{array}{c}\text { Counties within Atlanta COG } \\
\text { Buffer }^{1,2}(n=17)\end{array}$} & \multicolumn{3}{|c|}{$\begin{array}{c}\text { Counties within Augusta COG } \\
\text { Buffer }^{1,2}(n=8)\end{array}$} & \multicolumn{3}{|c|}{$\begin{array}{c}\text { Counties within Savannah COG } \\
\operatorname{Buffer}^{1,2}(n=4)\end{array}$} \\
\hline & $\begin{array}{l}\text { Counties in } \\
\text { 4th quartile } \\
\text { of } \mathrm{CC} \\
\text { incidence }^{3}\end{array}$ & $\begin{array}{c}\text { CC } \\
\text { Incidence } \\
\text { Rate }^{4,5}\end{array}$ & $\begin{array}{c}\% \\
\text { Uninsured }^{6}\end{array}$ & $\begin{array}{l}\text { Counties in } \\
\text { 4th quartile } \\
\text { of } C C \\
\text { incidence }^{3}\end{array}$ & $\begin{array}{c}\text { CC } \\
\text { Incidence } \\
\text { Rate }^{4,5}\end{array}$ & $\begin{array}{c}\% \\
\text { Uninsured }^{6}\end{array}$ & $\begin{array}{c}\text { Counties in } \\
\text { 4th quartile } \\
\text { of } \mathrm{CC} \\
\text { incidence }^{3}\end{array}$ & $\begin{array}{c}\text { CC } \\
\text { Incidence } \\
\text { Rate }^{4,5}\end{array}$ & $\begin{array}{c}\% \\
\text { Uninsured }^{6}\end{array}$ & $\begin{array}{c}\text { Counties in } \\
\text { 4th quartile } \\
\text { of } \mathrm{CC} \\
\text { incidence }^{3}\end{array}$ & $\begin{array}{c}\mathrm{CC} \\
\text { Incidence } \\
\text { Rate }^{4,5}\end{array}$ & $\begin{array}{c}\% \\
\text { Uninsured }^{6}\end{array}$ \\
\hline & $\mathrm{n}(\%)$ & $\underset{\mathrm{SD}^{7}}{\operatorname{mean}} \pm$ & $\underset{\mathrm{SD}^{7}}{\operatorname{mean} \pm}$ & $\mathrm{n}(\%)$ & $\underset{\mathrm{SD}^{7}}{\operatorname{mean}} \pm$ & $\underset{\mathrm{SD}^{7}}{\operatorname{mean}} \pm$ & $\mathrm{n}(\%)$ & $\underset{\mathrm{SD}^{7}}{\operatorname{mean}} \pm$ & $\underset{\mathrm{SD}^{7}}{\operatorname{mean}} \pm$ & $\mathrm{n}(\%)$ & $\underset{\mathrm{SD}^{7}}{\operatorname{mean}} \pm$ & mean $\pm \mathrm{SD}^{7}$ \\
\hline $\begin{array}{l}\text { Both } \\
\text { Races }\end{array}$ & $33(25.4)$ & $\begin{array}{c}14.96 \pm \\
5.85\end{array}$ & $10.28 \pm 1.86$ & $3(17.6)$ & $\begin{array}{l}16.47 \pm \\
2.23\end{array}$ & $10.78 \pm 2.83$ & $2(25)$ & $\begin{array}{c}12.11 \pm \\
7.20\end{array}$ & $10.88 \pm 2.15$ & $2(50)$ & $\begin{array}{c}17.3 \pm \\
1.80\end{array}$ & $10 \pm 2.79$ \\
\hline White & $34(26.2)$ & $\begin{array}{c}16.72 \pm \\
10.15\end{array}$ & --- & $1(5.9)$ & $\begin{array}{c}17.6 \pm \\
2.72\end{array}$ & --- & $3(37.5)$ & $\begin{array}{l}15.11 \pm \\
9.46\end{array}$ & --- & $1(25)$ & $\begin{array}{c}19.72 \pm \\
1.04\end{array}$ & --- \\
\hline Black & $27(20.8)$ & $\begin{array}{c}11.21 \pm \\
18.96\end{array}$ & --- & $10(58.8)$ & $\begin{array}{c}17.77 \pm \\
8.60\end{array}$ & --- & $1(12.5)$ & $\begin{array}{l}9.11 \pm \\
6.55\end{array}$ & --- & $0(0)$ & $\begin{array}{c}12.9 \pm \\
2.39\end{array}$ & --- \\
\hline
\end{tabular}

${ }^{1} \mathrm{COG}=$ childhood oncology group.

${ }^{2}$ The COG Buffer is a 40-mile radius surrounding the COG treatment facility.

${ }^{3}$ The $4^{\text {th }}$ quartile of CC incidence is the highest CC incidence rate quartile observed, varies by race.

${ }^{4} \mathrm{CC}=$ childhood cancer.

${ }^{5}$ Incidence rates were directly standardized age-adjusted per 100,000 population.

${ }^{6}$ Percentages of uninsured children (ages $<19$ ) were obtained from the 2011 US Census Bureau's SAHIE program.

${ }^{7} \mathrm{SD}$ : standard deviation. 
When access to care data (i.e., percent of uninsured children and COG buffer zones) were geographically overlaid onto the $\mathrm{CC}$ incidence rate maps, $\mathrm{CC}$ incidence rates were lower among children living within the Augusta COG buffer compared to those that live within any other COG buffer (Table 2). In the Atlanta COG buffer, 10 out of 17 counties $(58 \%)$ had high CC incidence rates for Black children (Table 2). Overall, Savannah had slightly higher CC incidence rates for White children compared to counties located in the other two COG buffers (Table 2). Rockdale County (located in north GA) was the only county that was identified as having a high percentage of uninsured children
(10.3\%), categorized in the highest quartile for CC incidence $(18.7$ per 100,000$)$ and was within the Atlanta COG buffer (Fig. 7). When stratified by race, the number of counties with uninsured children in the highest quartile (11.7\% - 19.1\% uninsured) and the highest quartile for CC incidence rates were 0 and 2 for White and Black children with cancer, respectively (Fig. 8; Fig. 9). Rockdale and Coweta Counties were the two counties identified in the Atlanta COG buffer as having a high percentage of uninsured children $(10.3 \%$ and $12.0 \%$, respectively) and having Black $\mathrm{CC}$ incidence rates in the highest quartile (45.2 and 16.3 per 100,000, respectively).

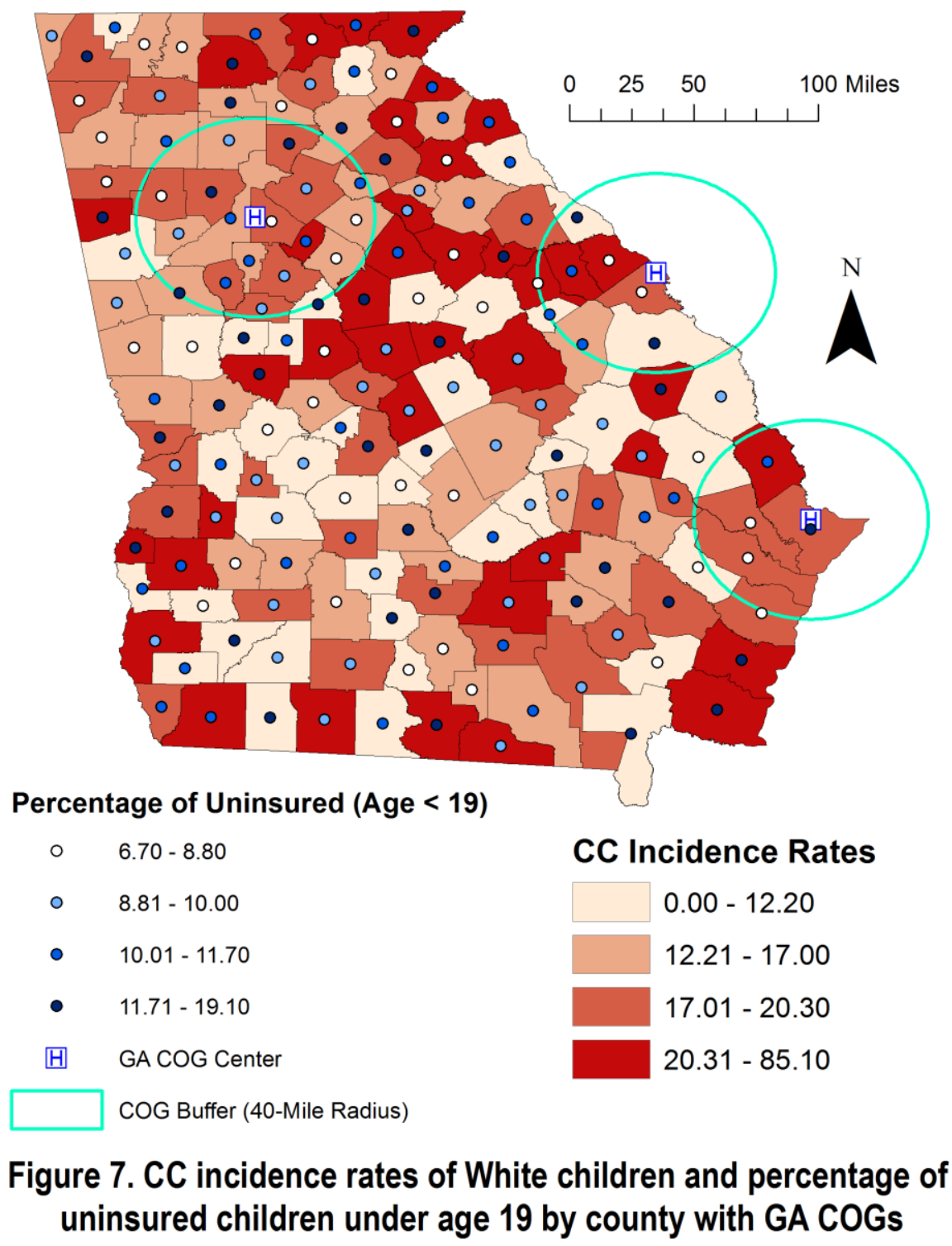




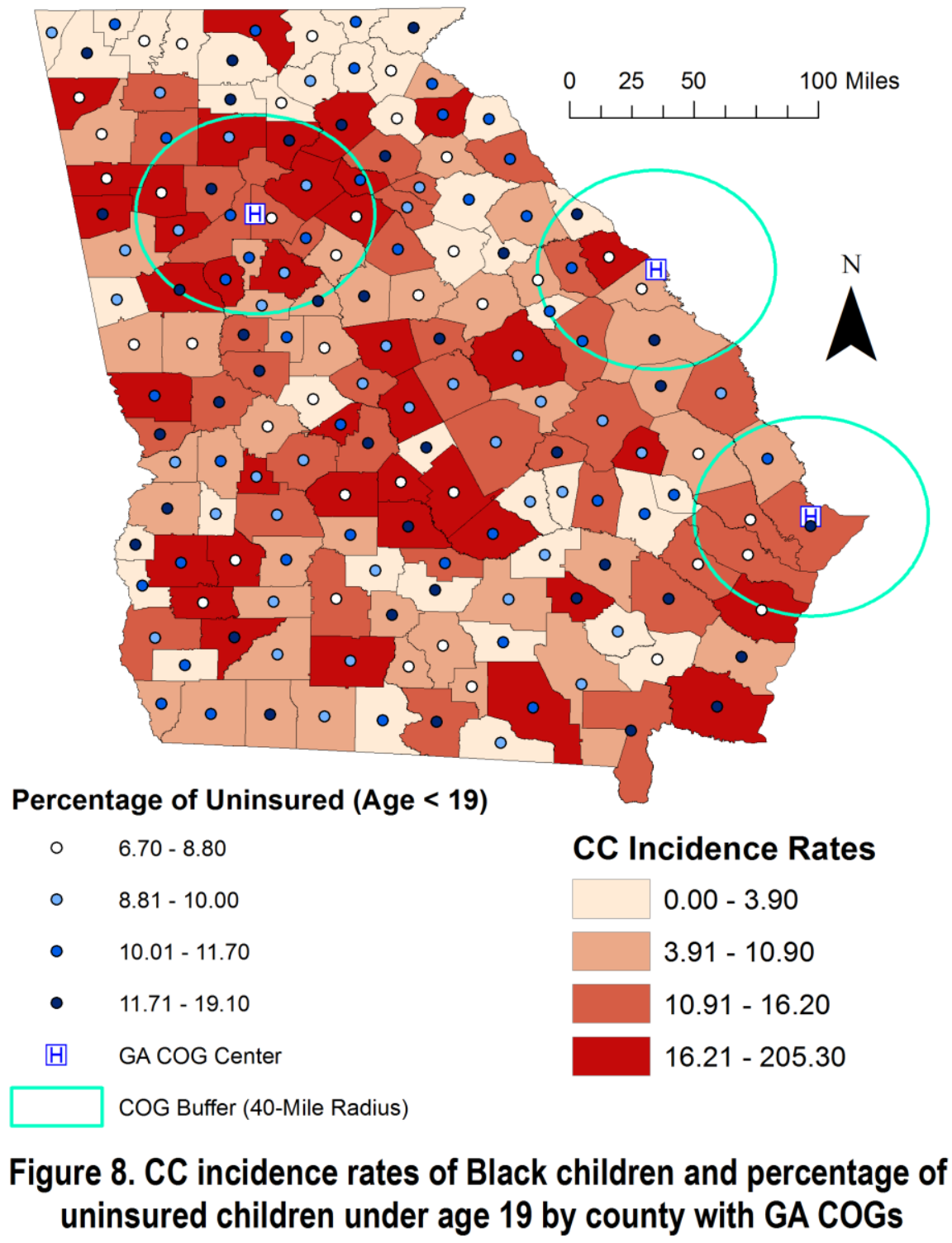

\section{DISCUSSION}

The purpose of this paper was to describe the epidemiology of $\mathrm{CC}$ in the state of $\mathrm{GA}$, by examining geographic trends by county, sex, and race and to explore potential disparities in health care access. The average CC incidence rate in GA from 2000-2011 was 15.7 cases per 100,000 children for all cancer types, slightly lower than the national CC incidence rate of 17.0 cases per 100,000 children for all cancer types. Although the overall CC incidence rate in GA was slightly lower than corresponding national rates, $36 \%$ of GA counties $(n=58)$ had CC incidence rates above the national average. White children in GA had significantly higher incidence rates for all cancer types, including leukemia and CNS cancers. This observation was consistent with national data on CC incidence (National Cancer Institute, n.d.). Additionally, leukemia was the cancer type with the highest incidence rates compared to all other cancer types in GA, followed by CNS cancers.
Access to health care as indicated by the percentage of uninsured children for each GA County and distance to COG centers (i.e., COG buffer) was explored in relationship to $\mathrm{CC}$ incidence rates. We found that about $25 \%$ of GA counties $(n=33)$ that were not within a COG buffer had children (ages < 19) who were uninsured (mean \pm SD: $10.28 \% \pm 1.86 \%$ ). The insurance coverage variable was of similar magnitude in counties included in the 40-mile radius COG buffer (mean \pm SD of all COG centers: $10.55 \% \pm$ $0.48 \%$ ). CC incidence varied geographically and in relation to the GA COG centers. Overall, counties within a 40-mile radius of the Augusta COG had lower CC incidence rates. Counties within a 40-mile radius of the Savannah and Atlanta COGs had higher $\mathrm{CC}$ incidence rates than all counties not falling inside a COG buffer. These descriptive findings suggest that there may be variability in the quality or accessibility of the COG centers, or, perhaps more likely, that populations residing in a closer proximity to each $\mathrm{COG}$ facility differ in potentially important CC predictors (e.g., 
socioeconomic status, education, lifestyle). Even though counties further away from COG centers did not have the highest $\mathrm{CC}$ incidence rates, they may have more severe outcomes (e.g., later stage at diagnosis, poorer survival), which were not assessed in the current study. Future studies should examine more refined data on population and tumor characteristics to fully characterize these variations.

High CC incidence rates and greater proportions of uninsured children (i.e., poorer access to care) overlapped in Rockdale County, located in the north portion of GA. According to the 2014 US census, Rockdale County had a population that is $45.9 \%$ White (U.S. Census Bureau, 2015d) and is about 34 miles away from the nearest certified pediatric oncology treatment center located in Atlanta, GA. This finding may suggest that children living with cancer in Rockdale County may represent a specific population of concern and future analytic or public health education programs may target this geography to improve outcomes.

Ensuring that children with cancer have adequate access to health care provides them with the greatest chance of survival (McDavid, Tucker, Sloggett, \& Coleman, 2003). Type of care (pediatric treatment center versus adult treatment center) is critical in terms of survival among children and adolescents living with cancer. Adolescents with cancer treated with a pediatric treatment plan at a pediatric treatment facility have a better chance of survival than adolescents with cancer not treated at a pediatric treatment facility (Howell et al., 2007). There is a delay in the diagnosis of cancer in adolescents who are uninsured compared to their insured counterparts (Martin et al., 2007). This delay can lead to late diagnoses of cancer and ultimately lower survival rates among this age group, further emphasizing the importance of age-appropriate treatment facilities and equitable health care access. It is important that a qualified team of experts, specifically trained in pediatric oncology, organizes treatment regimens in order to ensure that each child with cancer has the best probability of survival. In order to appropriately advocate for children with cancer living in GA, we need to work toward ensuring that children and adolescents with cancer are receiving ageappropriate treatment plans at a treatment facility that specializes in doctoring young patients.

COG treatment facilities are a network of hospitals meeting National Cancer Institute quality assurance metrics. These centers are hospitals or units within hospitals with oncology health care teams that specialize in treating children and adolescents with cancer. According to the Children's Oncology Group, there are three COG treatment facilities in GA: The Backus Children's Hospital at Memorial University Medical Center in Savannah, GA, Children's Healthcare of Atlanta Aflac Cancer Center in Atlanta, GA, and Children's Hospital of Georgia in Augusta, GA (Children's Oncology Group, 2014). These COG treatment facilities are located in three metropolitan areas in GA (U.S. Census Bureau, 2015f), leaving children who do not live close to these areas with the option to travel a great distance to a pediatric center or to be treated an a non-pediatric cancer center, which may result in poorer outcomes (Stock et al., 2008).
In the states surrounding GA, Florida (FL), South Carolina (SC), Alabama (AL), and Tennessee (TN), the distribution of COG centers vary. FL has 15 COG designated hospitals located within the state and is second to California, the state with the most COG facilities (19 COGs). Three COG facilities are distributed evenly throughout SC. AL has two COG centers located in the north and south region of the state. In $\mathrm{TN}$, four COG centers are located in the largest cities across the state and are associated with large hospitals or universities. It is important to note that Memphis, TN houses St. Jude's Children's Research Hospital, a nonprofit tertiary care cancer center that accepts patients regardless of financial or insurance status (Pui et al., 2012). Therefore, many children with cancer, regardless of their home state, seek treatment at St. Jude's Children's Research Hospital. The COG facilities in the states surrounding GA provide another option for families who live too far from COG centers within the state. The amount of COG treatment facilities in a state seems dependent on the size of the state rather than the percentage of children under the age of 18 . According to 2013 US census, GA and the states bordering GA have a similar percentage of the population under 18, ranging from $20.6 \%$ (FL) to $24.9 \%$ (GA) (U.S. Census Bureau, 2015b, 2015c). However, the total population of each state varies greatly, ranging from 4,771,929 (SC) to 19,600,311 (FL) (U.S. Census Bureau, 2015b, 2015c).

One strength of our study is that it is the first of its kind to provide a comprehensive overview of $\mathrm{CC}$ in GA, which included an evaluation of geographic trends in $\mathrm{CC}$ incidence and health care access disparities in the state. Our analysis suggests that children living in counties closest to $\mathrm{COG}$ facilities may still be disproportionally burdened by $\mathrm{CC}$, making additional examination of risk factors in these counties important. Additionally, there were significant geographical differences in $\mathrm{CC}$ incidence rates observed between White and Black children in GA. Knowledge of these underlying racial disparities in $\mathrm{CC}$ incidence may guide further research in examining risks in counties with a relatively large population of White children. Another strength of this analysis is its utilization of GA's gold certified cancer registry as its completeness facilitates an accurate description of incidence rates in this region.

Though further research is needed to fully comprehend the underlying explanations for these geographic and racial differences, this analysis provides preliminary support of exploring access to health care as a potentially important risk factor for higher $\mathrm{CC}$ incidence rates in specific GA counties. One restraint in our analysis is the lack of non-GA counties included in the Augusta and Savannah COG buffers. Our analysis focused on the state of GA but these COG buffers included surrounding SC counties. Another limitation to this study was that the access to care data readily available from the US Census Bureau's SAHIE program did not consider distance to age-appropriate treatment facilities as a measure of care. The observed geographic patterns in $\mathrm{CC}$ incidence likely represent the complex contribution of a variety of important risk factors. Although we explored access to care using a crude COG proximity buffer in relation to insurance status, no clear 
patterns were observed. Clearly, additional work is warranted and, to that extent, our team is continuing our research with the intention of using distance from case location to COG treatment facilities versus non-COG treatment facilities as a more refined measure of access to health care.

\section{Implications for Public Health}

This paper provides an overview of $\mathrm{CC}$ in the state to generate hypotheses about potential risk factors associated with geographic patterns in CC incidence. Specifically, we observed variation in $\mathrm{CC}$ incidence rates in counties near COG's, with the highest CC incidence rates observed near the Atlanta and Savannah COG's. Examining geographic patterns in $\mathrm{CC}$ incidence among this age group will identify areas of GA that may benefit from targeted education concerning the best age-appropriate treatment for this vulnerable population. Lower health quality and poor access to care is directly related to cancer mortality and survival. Understanding access to care and its correlation with CC incidence will allow future endeavors to explore access to care and its influence on cancer diagnosis and cancer survivorship. In the long term, results from this study provide preliminary evidence to inform more detailed individual-level epidemiologic studies, with the ultimate goal of designing evidence-based interventions towards CC prevention.

\section{References}

American Cancer Society. (2015). American Cancer Society Winn-Dixie Hope Lodge-Atlanta. Retrieved June 23, 2014, from

http://www.cancer.org/treatment/supportprogramsservices/hopel odge/baltimore/atlanta-staying-with-us

American Childhood Cancer Organization. (2015). Childhood Cancer Statistics. Retrieved May 23, 2015, from http://www.acco.org/about-childhoodcancer/diagnosis/childhood-cancer-statistics/

Bleyer, A. (2002). Older adolescents with cancer in North America: deficits in outcome and research. Pediatric Clinics of North America, 49(5), 1027-1042.

Centers for Disease Control and Prevention. (2014). United States Cancer Statistics (USCS). Retrieved October 4, 2014, from http://apps.nccd.cdc.gov/uscs/childhoodcancerbyprimarysite.asp $\underline{x}$

Children's Oncology Group. (2014). Locations. from http://www.childrensoncologygroup.org/index.php/locations/

Georgia Department of Public Health. (n.d.). Georgia Comprehensive Cancer Registry. Retrieved March 15, 2015, from http://dph.georgia.gov/georgia-comprehensive-cancerregistry

Howell, D. L., Ward, K. C., Austin, H. D., Young, J. L., \& Woods, W. G. (2007). Access to pediatric cancer care by age, race, and diagnosis, and outcomes of cancer treatment in pediatric and adolescent patients in the state of Georgia. J Clin Oncol, 25(29), 4610-4615. doi: 10.1200/jco.2006.07.6992
Martin, S., Ulrich, C., Munsell, M., Taylor, S., Lange, G., \& Bleyer, A. (2007). Delays in cancer diagnosis in underinsured young adults and older adolescents. The Oncologist, 12(7), 816824.

McDavid, K., Tucker, T. C., Sloggett, A., \& Coleman, M. P. (2003). Cancer survival in Kentucky and health insurance coverage. Archives of Internal Medicine, 163(18), 2135-2144.

McNamara C, B. A., Ward KC, Young JL. (2012). Georgia Childhood Cancer Report: Georgia Department of Public Health, Georgia Comprehensive Cancer Registry.

National Cancer Institute. (2013). A Snapshot of Pediatric Cancers. Retrieved October 4, 2014, from http://www.cancer.gov/researchandfunding/snapshots/pediatric

National Cancer Institute. (2014a). Cancer in Children and Adolescents. Retrieved October 3, 2014, from http://www.cancer.gov/cancertopics/factsheet/SitesTypes/childhood

National Cancer Institute. (2014b). State Cancer Profiles. 2014, from http://statecancerprofiles.cancer.gov/map/map.withimage.php?1 $3 \& 015 \& 515 \& 00 \& 0 \& 01 \& 0 \& 1 \& 6 \& 0$ - results

National Cancer Institute. (n.d.). Surveillance, Epidemiology, and End Results Program. Overview of the SEER Program. Retrieved March 15, 2015, from http://seer.cancer.gov/about/overview.html

Pui, C.-H., Pei, D., Pappo, A. S., Howard, S. C., Cheng, C., Sandlund, J. T., . . Rubnitz, J. E. (2012). Treatment outcomes in black and white children with cancer: results from the SEER database and St Jude Children's Research Hospital, 1992 through 2007. Journal of Clinical Oncology, JCO. 2011.2040. 8617.

Stock, W., La, M., Sanford, B., Bloomfield, C. D., Vardiman, J. W., Gaynon, P., . . . Nachman, J. (2008). What determines the outcomes for adolescents and young adults with acute lymphoblastic leukemia treated on cooperative group protocols? A comparison of Children's Cancer Group and Cancer and Leukemia Group B studies. Blood, 112(5), 1646-1654.

U.S. Census Bureau. (2015a). Small Area Health Insurance Estimates (SAHIE). Model-based Small Area Health Insurance Estimates (SAHIE) for Counties and States. Retrieved June 23, 2015, from http://www.census.gov/did/www/sahie/

U.S. Census Bureau. (2015b). State and County QuickFact Florida. Retrieved May 25, 2015, from http://quickfacts.census.gov/qfd/states/12000.html

U.S. Census Bureau. (2015c). State and County QuickFacts. Retrieved May 23, 2015, from http://quickfacts.census.gov/qfd/states/13000.html

U.S. Census Bureau. (2015d). State and County QuickFacts Rockdale County, Georgia. Retrieved June 23, 2015, from http://quickfacts.census.gov/qfd/states/13/13247.html

U.S. Census Bureau. (2015e). State and County QuickFacts Beta. Retrieved May 23, 2015, from http://www.census.gov/quickfacts/table/PST045214/13007,1320 $\underline{1}$

U.S. Census Bureau. (2015f). State and County QuickFacts Table. Retrieved May 23, 2015, from http://www.census.gov/quickfacts/table/PST045214/1304000,13 $\underline{04204,1369000}$ 